\section{Pneumocystis pneumonia: immunosuppression, Pneumocystis jirovecii...and the third man}

\author{
Christophe Duboucher, Rosa Boggia, Gérard Morel, Monique Capron, \\ Raymond J. Pierce, Eduardo Dei-Cas and Eric Viscogliosi
}

The Review article by Thomas and Limper on Pneumocystis pneumonia (PcP) published in Nature Reviews Microbiology illustrates the biological insights that have been gained from the use of molecular tools and the more recent technique of observation ${ }^{1}$. In this Correspondence, we discuss recent findings concerning the prevalence of
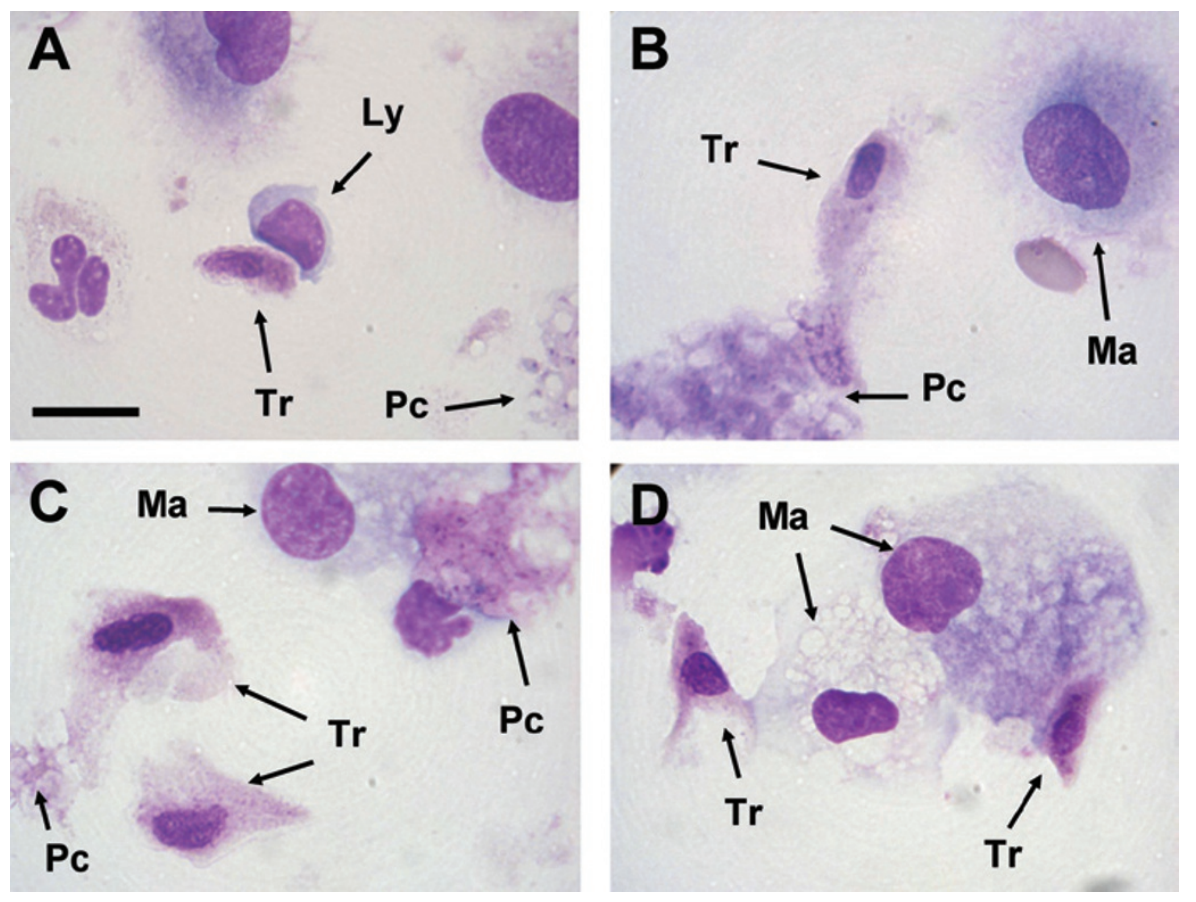

Figure 1 | Cytological appearance of trichomonads in a BALF sample taken from a patient with PcP. The figure is derived from an analysis of a bronchoalveolar lavage fluid (BALF) sample taken from a patient with Pneumocystis pneumonia (PcP) (an adult HIV-1-infected male). Trichomonad cells (Tr) can be identified in the vicinity of an aggregate of Pneumocystis (Pc) and macrophages (Ma). The stain used was May-Grünwald-Giemsa $\times 1,000$. The scale bar represents $10 \mu \mathrm{m}$. Other photomicrographs of samples taken from other patients with $\mathrm{PcP}_{\mathrm{C}}$ (from the same and other institutions) are available at http://christophe.duboucher.free.fr/trichomoniasis/. Ly, lymphocyte. been identified as belonging to the trichomonad group of protozoa (FIG. 2). These microorganisms multiply in alveoli and are found in BALF without oral or upperrespiratory-tract-fluid contamination. Furthermore, there is a strong correlation between the abundance of Pneumocystis organisms and trichomonad populations. It is established that Trichomonas tenax, a commensal of the human oral cavity, occurs in bronchial secretions during chronic respiratory conditions ${ }^{2,3}$. However, in the course of PcP, additional trichomonad species have been identified using molecular methods. These species include Trichomonas vaginalis, which is frequently found in the urogenital tract of humans ${ }^{4}$ and Tritrichomonas foetus, a genital trichomonad that is found in bovids ${ }^{5}$.

The recent observation of trichomonads in BALF samples during the course of acute respiratory distress syndrome (ARDS) suggests that, unlike other opportunistic infectious agents, the development of trichomonads in lungs is not directly linked to immunosuppression ${ }^{6}$. It is likely that $\mathrm{PcP}$ and ARDS generate similar local hypoxic conditions that favour the colonization and growth of microaerophilic trichomonads. Superinfection by trichomonads during PcP could, therefore, be a secondary event within alveolar lumina that has been obliterated by fungal pathogens; drugs active against PcP have consistently cured patients of pulmonary trichomonosis ${ }^{4,5}$.

From our numerous observations, trichomonads are frequently - if not always - found in the BALF samples of PcP patients ${ }^{7}$. Thus, the common disease called PcP, which is attributed exclusively to the extensive proliferation of Pneumocystis organisms, could, in fact, be a more complex process that involves trichomonad co-infection. By considering $\mathrm{PcP}$ as a co-infection, all the events that occur in alveoli, which until now have been attributed only to Pneumocystis, may have to be reconsidered. In BALF samples, trichomonads appear as amoeboid cells. Transformation into the amoeboid form results from the interaction of trichomonads 

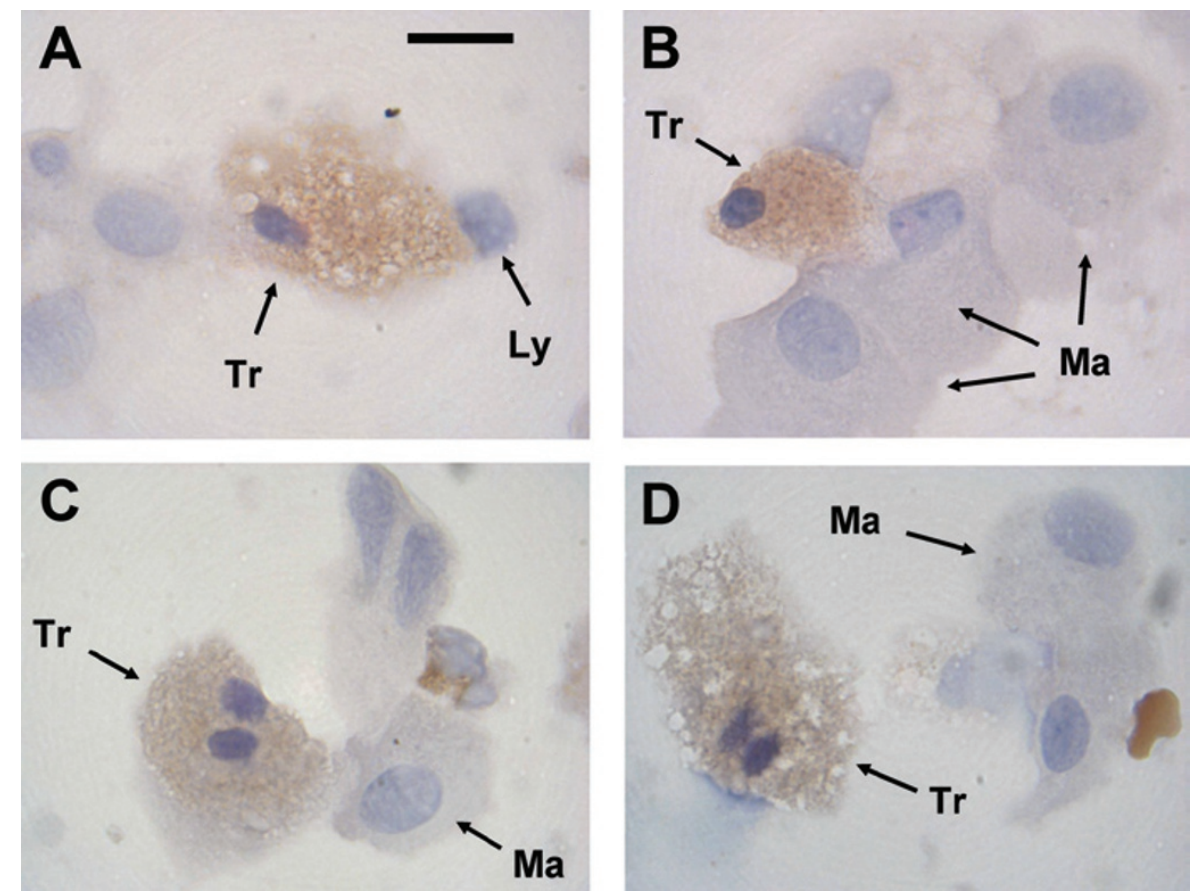

Figure 2 | Identification of trichomonads in a BALF sample taken from a patient with PcP using immunocytochemistry. The figure is derived from an analysis of a bronchoalveolar lavage fluid (BALF) sample taken from a patient with Pneumocystis pneumonia (PcP) (an adult HIV-1-infected male). Trichomonads were labelled by a commercially available monoclonal antibody that was produced against a surface antigen of Trichomonas vaginalis (clone 8.F.284; courtesy of US Biological, Massachusetts, USA). Trichomonads are coloured brown by the chromogen (diaminobenzidine). The scale bar represents $10 \mu \mathrm{m}$. Ly, lymphocyte; Ma, macrophages; Tr, trichomonad cells.

with host cells, which causes cytoskeletal changes, flagellum loss and the acquisition of pseudopodia. These amoeboid forms are deleterious to epithelial cells in vitro ${ }^{8,9}$. Moreover, T. vaginalis and T. foetus have been shown to be pathogenic to humans and bovids, respectively, and are able to induce apoptosis in epithelial cells and macrophages ${ }^{10-12}$. In addition, trichomonads are frequently observed in close contact with alveolar macrophages in BALF samples, which suggests that there is a direct interaction with host innate immune defences.

Together, these observations suggest that trichomonads have a potential pathogenic effect on the alveolar epithelium during the course of PcP disease. Even if the consequences of this parasitic superinfection remain hypothetical, the presence of trichomonads in the lungs of patients with PcP should not be considered to be merely anecdotal, and more attention should be given to these parasites.
Christophe Duboucher and Rosa Boggia are at the Department of Pathology, Poissy/Saint-Germain Hospital, Saint-Germain-en-Laye 78100, France.

Gérard Morel is at The Unité Mixte CNRS-University, Claude Bernard 5123, Villeurbanne 69622, France.

Monique Capron, Raymond J. Pierce and Eric Viscogliosi are at The Inserm U547, Lille Pasteur Institute \& Lille 2 University, Lille 59019, France.

Eduardo Dei-Cas is at Department of Ecology of Parasitism, Lille Pasteur Institute \& Lille 2 University Hospital Centre, Lille 59019, France.

Correspondence to C.D. e-mail: christophe.duboucher@online.fr

1. Thomas, C. F. Jr \& Limper, A. H. Current insights into the biology and pathogenesis of Pneumocystis pneumonia. Nature Rev. Microbiol. 5, 298-308 (2007).

2. Hersh, S. M. Pulmonary trichomoniasis and Trichomonas tenax. J. Med. Microbiol. 20, 1-10 (1985).

3. Mallat, H. et al. Molecular characterization of Trichomonas tenax causing pulmonary infection. J. Clin. Microbiol. 42, 3886-3887 (2004).

4. Duboucher, C. et al. Pulmonary co-infection by Trichomonas vaginalis and Pneumocystis sp. as a novel manifestation of AIDS. Hum. Pathol. 34, 508-511 (2003).

5. Duboucher, C. et al. Molecular identification of Tritrichomonas foetus-like organisms as coinfecting agents of human Pneumocystis pneumonia. J. Clin. Microbiol. 44, 1165-1168 (2006).

6. Duboucher, C. et al. Pulmonary superinfection by trichomonads in the course of Acute Respiratory Distress Syndrome. Lung 185, 295-301 (2007).

7. Duboucher, C. et al. Frequency of trichomonads as coinfecting agents in Pneumocystis pneumonia. Acta Cytol. 49, 273-277 (2005).

8. Arroyo, R., Gonzalez-Robles, A., Martinez-Palomo, A. \& Alderete, J. F. Signalling of Trichomonas vaginalis for amoeboid transformation and adhesion synthesis follows cytoadherence. Mol. Microbiol. 7, 299-309 (1993).

9. Jesus, J. B. et al. Trichomonas vaginalis virulence against epithelial cells and morphological variability: the comparison between a well-established strain and a fresh isolate. Parasitol. Res. 93, 369-377 (2004).

10. Sommer, U. et al. Identification of Trichomonas vaginalis cysteine proteases that induce apoptosis in human vaginal epithelial cells. J. Biol. Chem. $\mathbf{2 8 0}$ 23853-23860 (2005)

11. Singh, B. N. et al. Tritrichomonas foetus induces apoptotic cell death in bovine vaginal epithelial cells. Infect. Immun. 72, 4151-4158 (2004).

12. Chang, J. H. et al. Apoptosis of macrophages induced by Trichomonas vaginalis through the phosphorylation of p38 mitogen-activated protein kinase that locates at downstream of mitochondria-dependent caspase activation. Int. J. Biochem. Cell. Biol. 38, 638-647 (2006). 